\title{
A GaAs MESFET IC for Optical Multiprocessor Networks
}

\author{
JOHN D. CROW, MEMBER, IEEE, C. J. ANDERSON, S. BERMON, A. CALLEGARI, \\ JOHN F. EWEN, MEMber, IEEE, J. D. FEDER, J. H. GREINER, E. P. HARRIS, PETER D. HOH, \\ H. J. HOVEL, J. H. MAGERLEIN, T. E. MCKOY, A. T. S. POMERENE, \\ DENNIS L. ROGERS, MEMBER, IEEE, G. J. SCOTT, M. THOMAS, G. W. MULVEY, \\ B. K. KO, TOKEO OHASHI, MEMBER, IEEE, M. SCONTRAS, AND D. WIDIGER
}

\begin{abstract}
A GaAs, E/D mode, self-aligned, refractory-gate, MESFET chip process and circuit family has been developed for the integration of fiber-optic data link functions (e.g., photodetection, amplification, clock recovery, and deserialization) on a single chip. This paper describes the process and presents results on integrating a complete optical receiver, including the photodiode and clock recovery circuits, onto one chip. The chip functions use over 2000 devices, and perform at 1-Gbit/s speed, while dissipating less than $300 \mathrm{~mW}$ of heat. This chip is the most complex high-performance OEIC reported to date.
\end{abstract}

\section{INTRODUCTION}

$\mathrm{T}$ $\mathrm{HE}$ interconnection of many processors is a growing theme in the data processing industry [1], [2]. For optical data links to play a role in the interconnection networks of these multiprocessor complexes, a high-performance reliable, compact opto-electronic integrated circuit (OEIC) technology is needed to integrate network functions together on a single semiconductor chip [3]. Typical functions implemented at network interfaces, which would be important to capture on a chip are shown in Fig. 1, and include a mix of digital and analog, and optical and electronic functions, with a chip complexity at the LSI level. With powerful processors at the network nodes, multigigabit-per-second speed may be required in this chip (for example, $[4]^{1}$ ). The distances are likely to be short (less than $1 \mathrm{~km}$ ) to keep data transit times between the nodes short with respect to the machine instruction cycle time.

This paper describes a GaAs MESFET IC process that has many of the characteristics desired for this application. At this time, it appears that GaAs is the best technology candidate, as other implementations (e.g., based on Si or InP material systems) will be limited to bulky hybrid chip modules, low levels of integration, and yield

Manuscript received June 27, 1988; revised September 26, 1988.

J. D. Crow, C. J. Anderson, S. Bermon, A. Callegari, J. F. Ewen, J. D. Feder, J. H. Greiner, E. P. Harris, P. D. Hoh, H. J. Hovel, J. H. Magerlein, T. E. McKoy, A. T. S. Pomerene, D. L. Rogers, G. J. Scott, and $\mathrm{M}$. Thomas are with the IBM Thomas J. Watson Research Center, Yorktown Heights, NY 10598.

G. W. Muley, B. K. Ko, T. Ohashi, M. Scontras, and D. Widiger are with the IBM-GTD East Fishkill Facility, Hopewell Junction, NY 12533. IEEE Log Number 8825238.

${ }^{1}$ For example, the recently announced CRAY Y-MP/832 transfers data from RAM storage into an eight-processor "host" over channels at up to 1 Gbyte/s. and reliability concerns due to transistor limitations or complex strained layer epitaxial growth techniques [5], [6].

We also present experimental results on the integration of some key functions onto a single chip, performing at gigabit-per-second data transfer rates. The chip described represents the highest level of integration yet reported in integrated gigabit-per-second opto-electronics chips.

\section{MESFET OEIC}

Refractory-gate MESFET processes have gained favor for GaAs logic and memory applications because they are simple, provide good thermal stability of device characteristics, and offer potential for the reduction of device parasitic resistance and capacitance [7]. The self-aligned gate MESFET technology employed in fabricating the chip reported here is an enhancement of that described previously by some of the authors [8], with significant changes in lithography and wiring, and the incorporation of gate sidewalls with a moderate implant beneath them. The process employs ${ }^{47} \mathrm{SiF}^{+}$channel and source-drain implants, sputtered $\mathrm{WSi}_{0.11}$ refactory gates, $\mathrm{Ni}-\mathrm{Au}-\mathrm{Ge}$ ohmic contacts, $\mathrm{Si}_{3} \mathrm{~N}_{4}$ and $\mathrm{SiO}_{2}$ insulation, and $\mathrm{Ni}-\mathrm{Au}$ wiring. A gate length of $1 \mu \mathrm{m}$ was used in all circuits. The FET channel and photodetector implant was activated by furnace annealing either in an arsenic overpressure or with a $\mathrm{SiN}_{x} \mathrm{O}_{y}$ cap deposited by plasma-enchanced chemical vapor deposition (PECVD). Furnace annealing of the source-drain implants was carried out with a $\mathrm{Si}_{3} \mathrm{~N}_{4}$ cap. Following patterning of the gate and detector electrodes, a moderate-dose self-aligned implant was carried out, after which a blanket $\mathrm{Si}_{3} \mathrm{~N}_{4}$ layer was deposited and etched to leave triangular sidewalls at the edge of the gate. The sidewalls keep the heavy source-drain implant away from the gate edge, resulting in a reduction in gate capacitance and an improvement in breakdown voltage.

The lithography for these circuits, which have 11 masking levels, was carried out entirely by step-and-repeat projection lithography. Positive photoresists were used except for the gate and wiring levels. For these levels, a negative-tone image-reversed structure was used for optimum resolution (for the gate etch), and for retrograde 

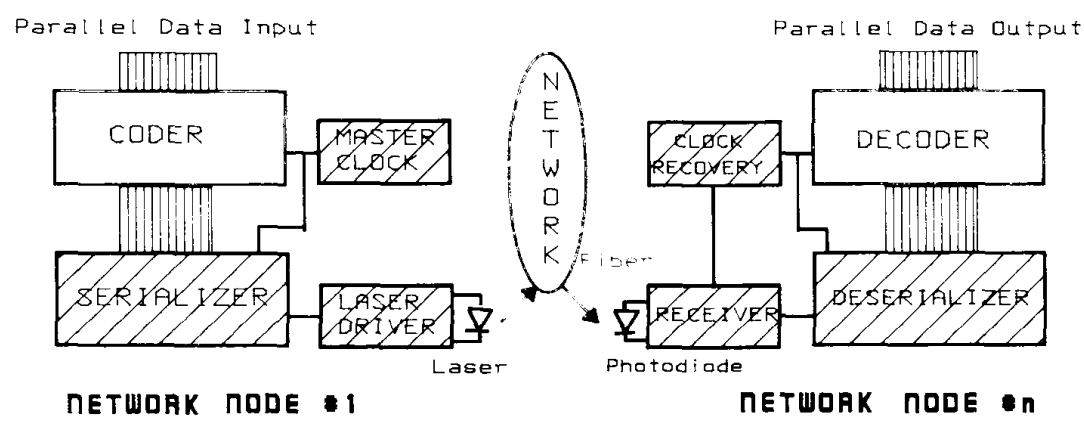

Fig. 1. Network interface functions: The typical functions required for an interface to a network. For full duplex operation. both sets of functions would be required at each network node. The shaded functions are performed at the bit speed of the serial link and thus require higher pertormance circuits

TABLE I

DIEVICF PARAMETLRS AFTHR COMPLITION OF WIRING AND AFTER ANNEALING AT $375^{\circ} \mathrm{C}$ FOR 1 AND $2 \mathrm{~h}$ (The values are averages of the sites on a typical 2 -in wafer.)

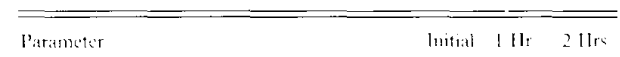

\begin{tabular}{|c|c|c|c|}
\hline 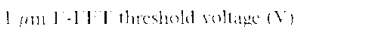 & $01 \times 1$ & 0.150 & (6). 1501 \\
\hline 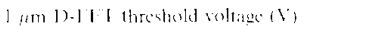 & $-11.5 ! t_{i}$ & -11.544 & -10.541 \\
\hline 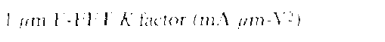 & (1) 17,1, & 11.158 & 11.157 \\
\hline 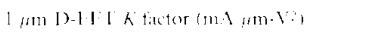 & 0.133 & 10.126 & $11.12^{7}$ \\
\hline 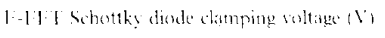 & 11.718 & 0.7114 & 11.7111 \\
\hline 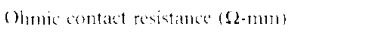 & 11.1 .34 & 10.296 & 4.353 \\
\hline 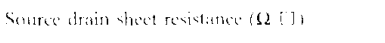 & 345 & 3.49 & $3.4 \%$ \\
\hline 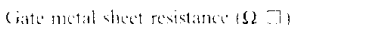 & 6.16 & 6.16. & 6.15 \\
\hline 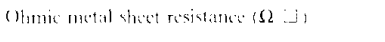 & 1.15 & 1.22 & 1.211 \\
\hline 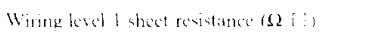 & $11.0 k$ & $11.1 \%$ & 01111 \\
\hline
\end{tabular}

lift-off profiles (used in the wiring levels). Although the optoelectonic circuits reported here only used $1.0-\mu \mathrm{m}$ gates, isolated $0.5-\mu \mathrm{m}$ gates have also been demonstrated with this process, indicating an even higher speed potential for these circuits.

Circuits of this level of integration require two full levels of global wiring in addition to $\mathrm{t}^{\circ} \mathrm{e} \mathrm{Ni-Au-Ge}$ contact metal. The first and second level wiring films consist of 500 and $700 \mathrm{~nm}$ of Au, respectively, with $10 \mathrm{~nm}$ of $\mathrm{Ni}$ at the bottom to improve film adhesion. Three insulating films deposited by PECVD are used. Generally the first level of insulation is $500 \mathrm{~nm}$ of $\mathrm{Si}_{3} \mathrm{~N}_{4}$ since its high tensile stress reduces the dependence of $V_{T}$ on the gate length and increases the FET $K$ factor. The second level of insulation is $700 \mathrm{~nm}$ of $\mathrm{SiO}_{2}$ used because of its lower dielectric constant and stress. Vias in the insulating films are formed by reactive-ion etching using a $\mathrm{CF}_{4} / \mathrm{O}_{2}$ process that gives an edge slope of about 45 degrees to allow good coverage by the successive metal film. An additional layer of PECVD $\mathrm{SiO}_{2}$ is deposited on top of the second layer of global metal for scratch protection and vias are etched at the pads.

The refractory-gate metallurgy, as well as the $\mathrm{Ni}-\mathrm{Au}$ Ge process with in-situ sputter clean used here [9], were chosen for their relatively good thermal stability. In order to access the stability of the metallurgy, wafers were tested after completion of the wiring levels, and then again after 1 and after $2 \mathrm{~h}$ of annealing at $375^{\circ} \mathrm{C}$. The results, which are summarized in Table I, suggest that all parameters remain within acceptable limits after annealing. The change in $V_{T}$ of about $30 \mathrm{mV}$ appears related to relaxation of the stress of the dielectric overlayer. FET's oriented at right angles to those reported here show a similar change in the opposite direction. No significant change in $V_{T}$ is observed during the second hour of annealing. The change in ohmic contact resistance for the circuit data shown in Table I was the closest to being unacceptable, and off-line experiments have indicated a way to lower these variations.

\section{Fully Integrated, Self-Clocked Optical Receiver OEIC}

We have used the above technology to fabricate a 1Gbit/s optical receiver containing the photodetector, amplifier, decision and bias control circuits, and clock recovery circuits necessary for an optical data link interface. A photo of this chip is shown in Fig. 2. These circuits have been described separately elsewhere [10]. [11], so their performance will only be summarized, pointing out where the above chip devices and processes aided functional performance. This paper is the first report of the combined operation of these functions on a single chip. These functions are the most noise and jitter sensitive functions in the link, as well as being a mix of analog and digital circuits, and therefore are a good testbed of the requirements for an OEIC. The total device count on the chip is $\sim 2500$, making it the most complex OEIC chip yet reported. It dissipates $\sim 300 \mathrm{~mW}$ of heat.

\section{A. Optical Receiver}

The receiver schematic is shown in Fig. 3, and consists of an interdigitated, metal-semiconductor-metal, 


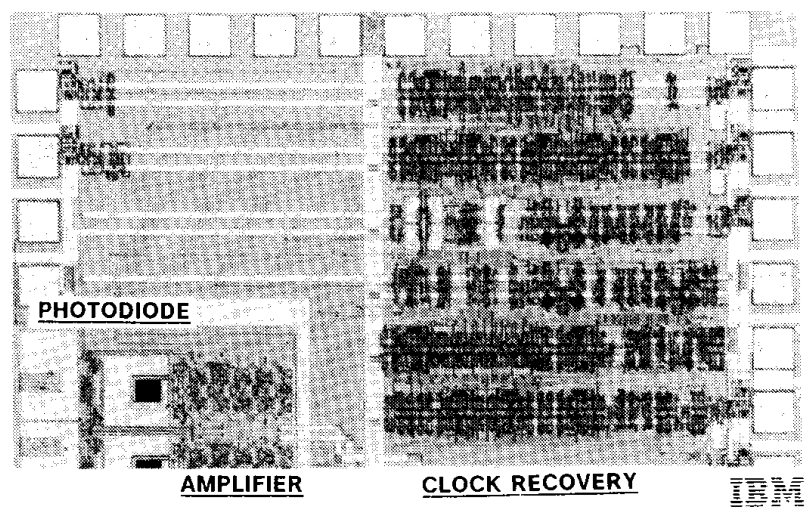

Fig. 2. Photo of the receiver/clock recovery portion of the chip. The space occupied by this much function is $-3 \mathrm{~mm}^{2}$.

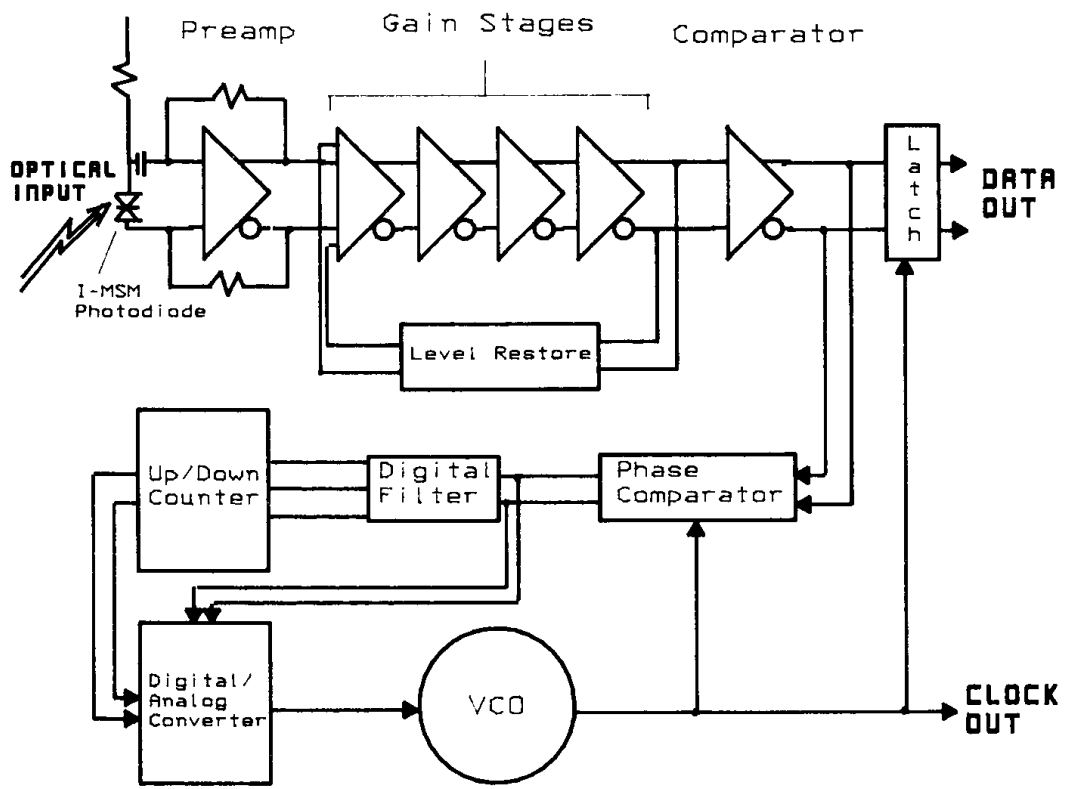

Fig. 3. Schematic of the receiver/clock functions: These are the functional blocks in the integrated receiver/clock. References [10], and [11] contain a more complete description of these circuits.

Schottky-barrier (I-MSM) photodiode, a transimpedance preamplifier, a four-stage, differential cascode-type amplifier, a Schmitt trigger decision circuit, and an automatic bias control circuit for the preamplifier. The IMSM detector utilizes the WSi gate metal because of its opitmal Schottky-barrier height, and to assure a temperature and time stable Schottky barrier for its contact fingers. The enchancement implant of the MESFET process was used under the detector to create a region that prevents surface trapping of photogenerated carriers, and thus prevents tails in the PD pulse response. The resulting dark current is 5 $\mathrm{nA}$, and the measured (by optical picosecond probing technique) optical bandwidth is $14 \mathrm{GHz}$. The active area of the detector was about $5.5 \times 10^{-5} \mathrm{~cm}^{2}$ making it useful with multimode fibers. Low input capacitance to the amplifier is important for high-sensitivity receivers. The
IMSM PD design yields a low PD capacitance of $150 \mathrm{fF}$, while the ability to integrate the detector close to the preamp yield a parasitic capacitance of $<20 \mathrm{fF}$. The sensitivity at $1 \mathrm{Gbit} / \mathrm{s}$ has been measured to be $-22 \mathrm{dBm}$ for an NRZ code and $B E R<10^{-9}$. The modeled receiver bandwidth is $>1.5 \mathrm{GHz}$ with a $-24-\mathrm{dBm}$ sensitivity, so that we expect a packaged chip to operate at over 2-Gbit/s speed.

The receiver circuit was designed to be entirely differential to improve its immunity to power supply noise and reduce its dependence on threshold voltage. This differential design and the use of a tight detector/amplifier coupling, possible only in a fully integrated design, resulted in little noise being coupled into the receiver input from other circuits on the same chip. Differential designs do require more transistors to implement, but a technology 


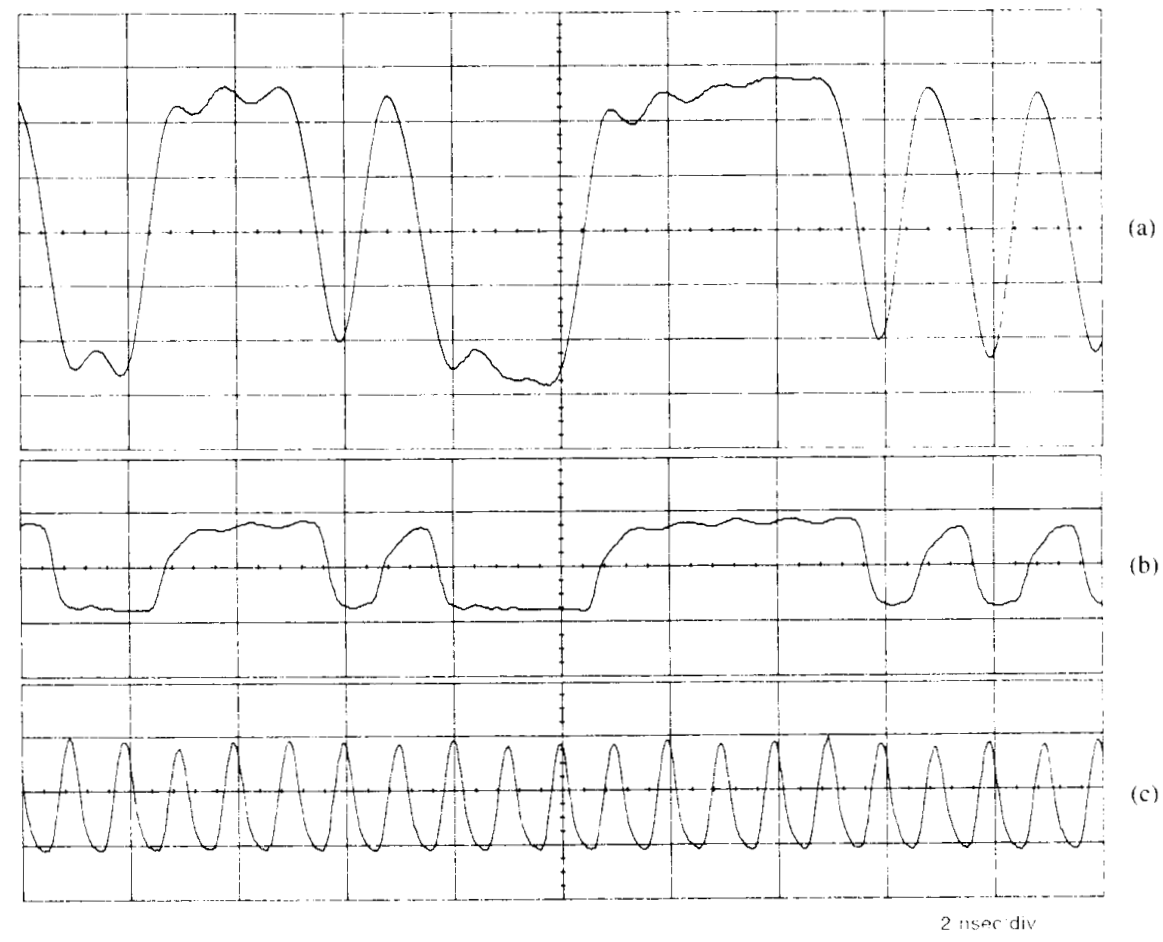

Fig. 4. Chip performance: Oscilloscope plot (a) shows a nominal input light level ( $-16 \mathrm{dBm})$ to the receiver (measured by a commercial high-speed photodiode); plot (b) is the data output; plot (c) is the recovered elock output. Horizontal scale is $2 \mathrm{~ns} / \mathrm{div}$.

with LSI IC capability means that transistors are a cheap commodity. This abundance of logic circuit capability becomes very important when designing real systems, which often have link monitoring and service functions to perform along with data transmission operations.

\section{B. Clock Recovery}

Fig. 3 also shows the schematic of the phaselock loop (PLL) clock recovery circuit. To make the chip less susceptible to noise, and take advantage of the digital characteristic of these MESFET transistors, digital circuits were utilized in the voltage-controlled ring oscillator (VCO) and the loop filter (a digital filter using up-down counters). Once again, differential circuits were employed in the PLL to prevent signal distortion and improve speed. The rms jitter measured is less than 25 ps at $1.25 \mathrm{Gbit} / \mathrm{s}$, and the locking range was \pm 10 percent of the bit rate.

\section{Fully Integrated Performance}

Fig. 4 shows typical operation of both clock and receiver on the same chip. Oscilloscope plot (a) is the 1.25 Gbit $/ \mathrm{s}, 2^{7}-1$ psuedorandom sequence input from a laser illuminating the receiver's photodiode (optical power nominally $-16 \mathrm{dBm}$ ). Oscilloscope plot (b) illustrates the recovered data output from the receiver, and (c) is the

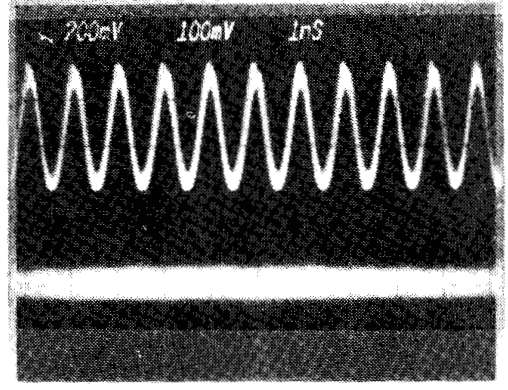

Free-running Clock

Fig. 5. Noise immunity of receiver: Oscillocope photo of receiver with no light input and free running clock on the chip operating at nominal bit rate. Top trace is clock signal output: bottom trace is receiver output. Horizontal scale is $1 \mathrm{~ns} /$ div. Vertical scale is arbitrary.

recovered clock. A 1/10-speed "byte clock" output is also available.

An important question associated with the integration of an optical receiver with large amounts of digital logic is the on-chip feedback from the digital circuits to the front end of the amplifier. Fig. 5 indicates the potential of excellent noise immunity arising from complete functional integration on a single chip. Shown in the oscilloscope trace is the receiver's amplifier when no optical input is illuminating the photodiode, taken while the clock on the chip is free-running at the bit speed, and driving a bit speed signal off the chip. On-chip noise would show up as a pattern effect at the output of the amplifier, and such patterns were not measureable. 


\section{Conclusion}

In conclusion, we have described an OEIC process and devices which are attractive to use in link adapters for optical multiprocessor networks. We have demonstrated a fully integrated optical receiver, with clock recovery, which is state of the art for integration complexity in highspeed OEIC chips. As the electronic technology is built on a digital LSI IC base, other digital circuits can be readily integrated onto this chip, and results will be reported elsewhere.

\section{ACKNOWLEDGMENT}

We acknowledge the processing and circuit design support from R. Dunne, S. Fang, D. Lacey, D. Piazza, J. Simmons, M. Su, and V. Tom. We also wish to acknowledge the support and encouragement of E. Vanderveer and M. Brodsky of our lab.

\section{REFERENCES}

[1] R. Bernard, "Computing at the speed limit," IEEE Spectrum, vol. 19, p. 26, Jan. 1984

[2] T. Manuel, "Parallel processing," Electronics, pp. 105-114, June 1983.

[3] J. W. Goodman, F. J. Leonberger, S.-Y. Kung, and R. A. Athale, "Optical interconnections for VLSI systems," Proc. IEEE, vol. 72, p. 850 , July 1984

14] T. Costlow, "Cray speeds up its Act with 6 nsec supercomputer," EE Times Newspaper, p. 4, Feb. 15, 1988

[5] S. R. Forrest, "Monolithic optoelectronic integration: A new component technology for lightwave communication," J. Lightwave Technol., vol. LT-3, p. 1248, Oct. 1985.

[6] A. Suzuki et al., "InGaAsP/InP long wavelength optoelectronic integrated circuit (OEIC's) for high-speed optical fiber communication systems," J. Lightwave Technol., vol. LT-5, p. 1479, Oct. 1987

[7] N. Matsunaga, M. Miyazaki, Y. Umemoto, J. Shigeta, H. Tanaka, and $\mathrm{H}$. Yanazawa, "Gallium arsenide MESFET technologies with 0.7 gate length for $4 \mathrm{~kb}, 1$ ns static RAM," in Proc. IEEE GaAs IC Symp., Oct. 1987, pp. 129-132.

[8] J. H. Magerlein et al., "Characterization of GaAs self-aligned, refractory gate, metal-semiconductor (MESFET) integrated circuits," J. Appl. Phys., vol. 61, p. 3080, Apr. 1987

[9] A. Callegari, E. T.-S. Pan, and M. Murakami, "Uniform and thermally stable AuGeNi ohmic contacts to GaAs," Appl. Phys. Lett., vol. 46, p. $1141,1985$.

[10] D. L. Rogers, "A fully integrated, $1 \mathrm{~Gb} / \mathrm{s}$, GaAs MESFET fiber optics receiver," in Proc. OFC'88, no. WF2, Jan. 1988.

[11] J. F. Ewen, "Fully integrated Gb/s clock recovery circuit in GaAs," in Proc. OFC' 88 , no. WQ19, Jan. 1988.

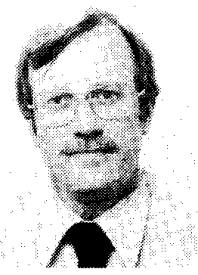

John D. Crow (S'68-M'78) received the B.S., M.S. and Ph.D. degrees in electrical engineering from the University of California, Berkeley, in 1966, 1968, and 1972 respectively.

He joined IBM's Research staff at the Thomas J. Watson Research Center in 1974, after two years of work on high-power propagation in optical fibers and photochromic planar lightguides at the Corning Glass Works Research Center. In 1979, he became Manager of the Optical Interconnect Technologies Group, working on highspeed computer links. His current project is to develop a gigabit-per-second fiber-optic link technology, utilizing GaAs OEIC chips.

Dr. Crow has received IBM Research Division Outstanding Contribution Awards for work on injection laser arrays for liquid-crystal displays in 1979, and for high-speed fiber-optic link development in 1983. In 1986 , he was on the technical program committee of the IEEE Optical Fiber Communication Conference. In 1987, he was appointed to the Photonics Panel of the U.S.-Government's National Research Council.
C. J. Anderson, photograph and biography not available at the time of publication.

S. Bermon, photograph and biography not available at the time of publication.

A. Callegari, photograph and biography not available at the time of publication.

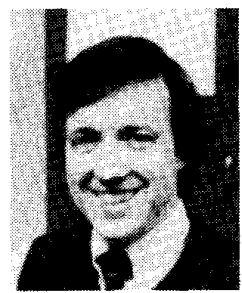

John F. Ewen (S'80-M'81) was born on November 11,1955 in Chicago, IL. He received the B.S.E.E., M.S.E.E, and Ph.D. degrees in electrical engineering from Purdue University, West Lafayette, IN, in 1976, 1977, and 1981, respectively. His graduate work dealt with surface acoustic wave devices and nonlinear acoustic wave propagation in lithium niobate and $\mathrm{ZnO} / \mathrm{Si}$ material systems.

In 1981, he joined the Fiber Optic Technologies group at the IBM Thomas $J$. Watson Research Center, Yorktown Heights, NY, where he has been involved in design and fabrication of high-speed optical links for computer applications, using both $\mathrm{Si}$ and $\mathrm{GaAs}$ technologies. He is currently developing high speed clock recovery circuits using GaAs MESFET technology for gigabitper-second optical data links.

Dr. Ewen is a member the American Physical Society.

J. D. Feder, photograph and biography not available at the time of publication.

J. H. Greiner, photograph and biography not available at the time of publication.

E. P. Harris, photograph and biography not available at the time of publication.

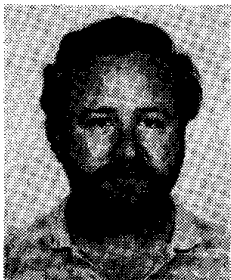

Peter $D$. Hoh received degrees in chemistry from Pace University and the University of Missouri.

$\mathrm{He}$ is currently on assignment for IBM at their Zurich, Switzerland, Research Laboratory. He is the author of numerous publications regarding semiconductor process technology and holds several U.S. and foreign patents.

H. J. Hovel, photograph and biography not available at the time of publication.

J. H. Magerlein, photograph and biography not available at the time of publication.

T. E. McKoy, photograph and biography not available at the time of publication. 
A. T. S. Pomerene photograph and biography not available at the time of publication.

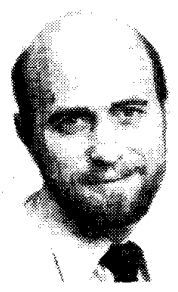

Dennis L. Rogers (M'83) recejved the B.A. degree in physics form California State University in 1968 and the M.A. and Ph.D. degrees in physics from the University of California in 1970 and 1978 , respectively.

Since then, he has been employed at the IBM Thomas J. Watson Research Center, Yorktown Heights, NY, as a Research Staff Member. His work includes the development of high-speed fiber-optic receivers and opto-electronic devices.

Dr. Rogers is a member of the American Physical Society.

G. J. Scott, photograph and biography not available at the time of publication.

\section{$*$}

M. Thomas, photograph and biography not available at the time of pub lication.
G. W. Mulvey, photograph and biography not available at the time of publication.

B. K. Ko, photograph and biography not available at the time of publica tion.

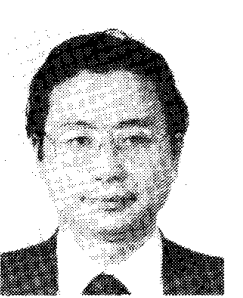

Takeo Ohashi (S'84-M-85) was born in Tokyo, Japan, in 1957. He received the B.S. degree in applied physics from the University of Tokyo in 1980 and the $\mathrm{Ph} . \mathrm{D}$. degree in electrical engineering from Cornell University in 1985

He joined the IBM East Fishkill Development Laboratory after graduating and has been involved with establishing gallium arsenide semiconductor technology.

M. Scontras, photograph and biography not available at the time of pub lication.

D. Widiger, photograph and biography not available at the time of publication. 